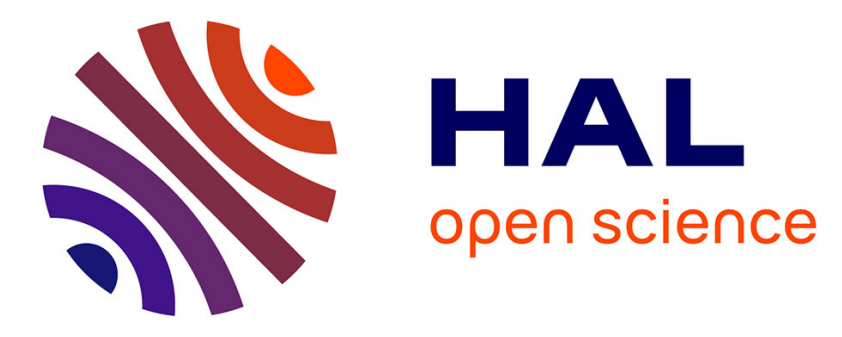

\title{
L'échange dans la documentation diplomatique bourguignonne: autour du vocabulaire des transferts
}

Eliana Magnani

\section{To cite this version:}

Eliana Magnani. L'échange dans la documentation diplomatique bourguignonne: autour du vocabulaire des transferts. Irmgard Fees, Philippe Depreux. Tauschgeschäft und Tauschurkunde vom 8. bis zum 12. Jh. / L'acte d'échange, du VIIIe au XIIe siècle, Böhlau-Verlag, pp.403-426, 2013, Beihefte zum Archiv für Diplomatik, Schriftgeschichte, Siegel- und Wappenkunde, Band 13, 978-3-412-21001-4. halshs-00703757v2

\section{HAL Id: halshs-00703757 https://shs.hal.science/halshs-00703757v2}

Submitted on 10 Nov 2013

HAL is a multi-disciplinary open access archive for the deposit and dissemination of scientific research documents, whether they are published or not. The documents may come from teaching and research institutions in France or abroad, or from public or private research centers.
L'archive ouverte pluridisciplinaire HAL, est destinée au dépôt et à la diffusion de documents scientifiques de niveau recherche, publiés ou non, émanant des établissements d'enseignement et de recherche français ou étrangers, des laboratoires publics ou privés. 


\section{L'échange dans la documentation diplomatique bourguignonne :} autour du vocabulaire des transferts

paru dans Tauschgeschäft und Tauschurkunde vom 8. bis zum 12. Jh. / L'acte d'échange, du VIII au XII siècle, Hg. Irmgard Fees, Philippe Depreux, Wein-Köln-Weimar, Böhlau-Verlag, 2013 (Beihefte zum Archiv für Diplomatik, Schriftgeschichte, Siegel- und Wappenkunde, Band 13), p. 403-426

(ISBN 978-3-412-21001-4)

Eliana MAGNANI

CNRS - Artehis - UMR 5594

eliana.magnani@u-bourgogne.fr

Nos concepts généraux sont encore instables et imparfaits Marcel Mauss ${ }^{1}$

Cette étude propose une approche préliminaire du vocabulaire des transferts à partir d'une masse importante de documents soumise au traitement du logiciel Philologic ${ }^{2}$. Il s'agit d'une étude expérimentale, du point de vue méthodologique et théorique, et qui s'appuie sur ce que Franco Moretti a appelé la « lecture à distance » (distant reading) par opposition à la « lecture de près » (close reading), c'est-à-dire, « où la distance n'est pas un obstacle mais une forme spécifique de connaissance », qui «permet de concentrer l'attention sur les unités qui sont beaucoup plus petites ou beaucoup plus grandes que le texte: formules, thèmes, tropes, ou genres et systèmes » ${ }^{3}$. Selon cette perspective, plutôt que les « actes d'échange » en tant qu'unité textuelle d'analyse, j'envisagerai les mots, les syntagmes ou les formules présents dans ce «genre» de texte du point de vue de leur fréquence et de leurs cooccurrences ${ }^{4}$.

\footnotetext{
${ }^{1}$ M. MAUSS, Rapports réels et pratiques de la psychologie et de la sociologie, in Journal de Psychologie Normale et Pathologique (1924), p. 922.

2 Logiciel libre développé par Mark Olsen et son équipe à l'Université de Chicago (http://philologic.uchicago.edu/). Je remercie vivement Alain Guerreau de m'avoir initiée à ce logiciel et, avec Nicolas Perreaux, de m'avoir aidée à son installation. Les travaux d'Alain Guerreau, constituent la principale référence en ce qui concerne l'analyse sémantique appliquée aux textes médiévaux, voir notamment, A. GuERREAU, L'avenir d'un passé incertain. Quelle histoire du Moyen Âge au XXI siècle ? (2001), p. 191-237 et ID., Le champ sémantique de l'espace dans la Vita de saint Maïeul (Cluny, début du XI ${ }^{\mathrm{e}}$ siècle), in Journal des savants (1997) p. 363-419.

${ }^{3}$ F. MoReTti, Conjectures on wolrd literature, in: New Left Review 1 (2000) p. 54-68 (ici p. 57, surligné par l'auteur) et F. MORETTI, Graphes, cartes et arbres. Modèles abstraits pour une autre histoire de la littérature, trad. fr. d'E. Dobenesque (2008) [Graphs, maps, trees: abstract models for a literary history (2005)].

${ }^{4}$ Une telle approche demanderait également une exploitation statistique des données, que nous ne sommes pas
} 
Le corpus pris en compte ici est constitué d'actes diplomatiques bourguignons édités antérieurs à 1300, réunis dans la base de données des CBMA - Chartae Burgundiae Medii $A e v i^{5}$ - que nous développons au sein d'Artehis UMR 5594, augmentés de quelques recueils de la vallée du Rhône et de la Provence, qui, avec la Bourgogne, forment une 'région diplomatique' dont certaines caractéristiques sont assez proches. Dans l'ensemble qui réunit un total de 14474 unités documentaires, il faut noter le poids quantitatif des 5574 chartes de l'abbaye de Cluny qui sont plus d'un tiers du total, et la concentration des actes, d'abord entre le milieu du $\mathrm{X}^{\mathrm{e}}$ et le milieu du $\mathrm{XI}^{\mathrm{e}}$ siècle (3984 actes - 27,5\%) et ensuite au XIII ${ }^{\mathrm{e}}$ siècle (3591 actes $-24,8 \%)^{6}$.

Les principaux termes désignant l'échange ont été interrogés à partir d'une série de troncatures -commut*, mutua*, permut*, transmut*, *cambi ${ }^{*}$, concami $^{*},{ }^{*}{ }_{\text {scami }}^{*},{ }^{*}$ xсатi ${ }^{*}$, *scang*, *schang* - qui permettent de rendre compte de la plupart des formes présentes, à partir, d'une part, du latin cicéronien commutare/commutatio dérivé, par préfixation de mutare/mutatio ainsi que permutare/permutatio, tous des termes au double sens de changement et d'échange ${ }^{7}$; et d'autre part du latin dérivé du gaulois cambiare qui est à l'origine des mots changer/échanger dans la plupart des langues latines anciennes et actuelles $^{8}$. Outre leur distribution chronologique, les formes ont été observées par rapport à leurs cooccurrences les plus fréquentes, dans la limite des cinq mots placés à leur gauche et à leur droite. Il s'agit là de l'un des principaux atouts offerts par Philologic. Nous avons pu ainsi dégager quelques tendances générales qui concernent et articulent entre eux, le champ

encore en mesure de réaliser ici, d'où le caractère expérimental de cet article.

${ }^{5} \mathrm{http}: / /$ www.artehis.eu/spip.php?article632\&var mode $=$ calcul

${ }^{6}$ Voir les tableaux 1 et 2 en annexe. En ce qui concerne les comptages d'actes et les répartitions chronologiques, donnés ici à titre indicatif, il faut noter, d'abord, qu'il s'agit des datations proposées par les éditeurs des actes, et ensuite que, dans le cas des datations approximatives par fourchette chronologique, Philologic, logiquement, prend en compte le terminus a quo, et c'est ce premier terme qu'on retrouvera indiqué dans les références des actes cités en note. Cela introduit, bien entendu des imprécisions inévitables, et appelle à la prudence dans l'interprétation des données, notamment dans les répartitions de plus courte durée, par décennies ou par quart de siècle, que nous avons testé mais pas retenues ici. Les tranches chronologiques plus larges, par siècle, voire demi-siècle, permettent d'atténuer davantage ces distorsions.

${ }^{7}$ Sur les différentes acceptions de commutatio, voir I. ROSE, Commutatio, le vocabulaire de l'échange chrétien au Haut Moyen Âge, in : Les élites et la richesse au Haut Moyen Âge, dir. J.-P. DEVROEY, L. FELLER, R. LE JAN (2010) p. 113-138, en particulier p. 119-127, sur l'enquête dans les CBMA.

${ }^{8}$ Pour plus d'agilité, nous avons limité la liste aux termes les plus courants tout en reconnaissant la grande diversité de ce champ sémantique. En effet, une simple recherche inversée dans la version électronique du Mediae latinitatis lexicon minus de Jan Frederik Niermeyer ( $1^{\mathrm{e}}$ éd. Leiden, E. J. Brill, 1976), montre que échange/échanger figurent comme traduction dans 45 entrées : alternare, alternatio, ambiare/angire/angiare, ammiare/amiare, cambire, cambitio, cambitus, cambium/camnium/camium/cambia/cambio, commercium, commutare, commutatio, commutator, complacitare, complacitatio, concambiare/camniare/camiare/cambire, concambiarius (-bita-), concambiatio, concambiator, concambium/concamnium/concamium, concamblaliter, conquestare, contra, decambiare, demutare, excambire (exqu-, esc-, esch-, sc-), ingrossare, inscontrum, meritum, mutuare, pariculus, precarius, procambiare, procambiatio, procambium, revestitor, servitium, talio, vicaneum, vicaria, vicariare, vicariatio/vicaratio/vicariacatio, vigoratio, xenium. 
sémantique des transferts, l'usage et la transformation des formulaires, les niveaux linguistiques, vernaculaire et 'technique'.

Les formes, nominales et verbales, désignant l'échange n'apparaissent pas seulement dans les «actes d'échange ». Comme pour les occurrences des mots, l'estimation du nombre d'actes du corpus faisant état d'une (ou plusieurs) action juridique considérée comme un échange est relativement faible, environ $5 \%$, contre $14 \%$ de ventes et, surtout, $65 \%$ de donations $^{9}$. Dans ce contexte bien connu, mais encore incomplètement expliqué, d'une prédominance des transferts désignés par le vocabulaire du don, la définition même des différents types de transferts reste posée. Cette étude entend aussi contribuer à ce débat qui dépasse, bien entendu, le seul cas de l'Occident médiéval.

En effet, par opposition à la société marchande contemporaine et à la suite des travaux de Marcel Mauss [1872-1950], il est devenu unanime de considérer les sociétés pré-modernes comme caractérisées par l'échange ${ }^{10}$, terme qui prend ainsi une acception plus large et lâche que l'échange en tant que troc. Comme l'a fait remarquer Alain Testart [1945-], cette superposition terminologique, et définitionnelle, n'est pas à même de donner un cadre théorique d'analyse clair, d'où sa proposition d'une définition de trois types de transferts, à partir du critère de l'exigibilité contraignante (juridique) de la contrepartie. Il faudrait ainsi appeler «don» le transfert dont la contrepartie ne peut pas être exigée, «échange » le transfert dont la contrepartie est exigible, et le «transfert du troisième type » le transfert exigible sans contrepartie ${ }^{11}$, auxquels François Athané [1973-] propose d'ajouter un quatrième type, la «prédation», transfert exigé sans être exigible ${ }^{12}$. En suivant cette définition d' « échange », ce que nous désignons habituellement par échange et vente/achat sont un même type de transfert. Ces travaux invitent ainsi à poser l'importante question du passage d'un type de transfert à l'autre (du don au dû ; de la prédation au tribut...) et de détecter des transferts « hybrides ». L'enjeu étant d'affiner les catégorisations, de repérer plus largement des régularités et des prévisibilités des transformations. L'étude de l'environnement lexical des transferts au Moyen Âge est l'une des approches à mettre en œuvre pour cette recherche, qui doit également prendre en compte le fait même de la mise par

\footnotetext{
${ }^{9}$ Sur les différentes formes de transaction à partir du cas de Cluny, voir B. RosEnwEIN, To Be the Neighbor of Saint Peter. The Social Meaning of Cluny's Property, 909-1049 (1989), p. 80-88 pour les échanges.

${ }^{10}$ En ce qui concerne l'héritage maussien au sein des études sur le Moyen Âge, je me permets de renvoyer à E. Magnani, Les médiévistes et le don. Avant et après la théorie maussienne, in: Don et sciences sociales. Théories et pratiques croisées, dir. E. MAGNANi (2007) p. 15-28.

${ }^{11}$ A. TeStART, Critique du don. Études sur la circulation non marchande (2007), en particulier ch. 2 : Les trois modes de transfert.

${ }^{12}$ F. Athane, Le don, histoire du concept, évolution des pratiques (2008) (thèse université Paris Ouest La Défense), thèse parue sous le titre Pour une histoire naturelle du don (2011).
} 
écrit, et plus largement de la mise en registre, écrite, graphique et iconique des transferts, à une échelle inédite, ce qui nous ramène aux observations de Jack Goody [1919-] sur les procédés d'écriture non seulement en tant que forme de stockage d'informations mais agissant à l'intérieur d'un « système structuré et structurant de rapports au monde ${ }^{13}$.

\section{De la commutatio à la permutatio, et la permanence de cambio}

A l'égard des données réunies en annexe dans les tableaux 1 et 2 et dans les graphiques 1 et 2 on peut faire une série de premières constatations. D'abord, le nombre d'actes contenant des formes relatives à l'échange suivent la tendance générale de l'ensemble des actes, avec une concentration au $\mathrm{X}^{\mathrm{e}}$ et $\mathrm{au} \mathrm{XIII}^{\mathrm{e}}$ siècle. Les occurrences les plus nombreuses dans l'ensemble du corpus concernent les mots en commut* $(849)^{14}$, suivies de ${ }^{*}$ cambi $^{*+}{ }^{15}(686)$ et de permut* (281); les occurrences de mutua* (69), recherchées car apparaissant souvent en association (synonymique ?) avec commutatio, concambio et scambio, et transmut* (16), étant plus ponctuelles. A l'inverse des formes issues de commutatio et de permutatio, dont la concentration est bien marquée du milieu du $\mathrm{IX}^{\mathrm{e}}$ au milieu du XI $\mathrm{I}^{\mathrm{e}}$ siècle, avec un pic au $\mathrm{X}^{\mathrm{e}}$ pour commut*, et à partir du milieu du XIII ${ }^{\mathrm{e}}$ pour permut*, les formes issues de cambio suivent le mouvement général des occurrences. Cette stabilité des formes issues de cambio, malgré leurs nombreuses variantes orthographiques, peut probablement être mise en rapport avec leur caractère vernaculaire. Les formes dérivées de commutatio, qui tendent à disparaître au profit des formes issues de permutatio au XIII ${ }^{\mathrm{e}}$ siècle, seraient en revanche, liées à un emploi lettré, technique. Permutatio est en effet le terme employé dans le droit romain pour désigner les échanges ${ }^{16}$, remis à l'usage au XIII ${ }^{\mathrm{e}}$ siècle au détriment, et on pourrait sans doute dire, à la place de commutatio. Il est aussi utilisé préférentiellement par les éditeurs du XIX ${ }^{\mathrm{e}}$ siècle pour désigner les actes d'échange (charta permutationis).

\section{Commutatio : formules et champ sémantique des transferts}

\footnotetext{
${ }^{13}$ J. Goody, La raison graphique. La domestication de la pensée sauvage, trad. et présentation de J. Bazin et A. Bensa (1979) [The Domestication of the Savage Mind (1977)].

${ }^{14}$ Les chiffres entre parenthèses indiquent le nombre d'occurrences. Les différentes formes et leurs fréquences sont données en annexe.

${ }^{15}$ Nous désignons ici $*$ cambi ${ }^{*+}$, la somme des occurrences des dérivés de cambiare $\left({ }^{*}\right.$ cambi $^{*}$, concami*, *scami*, *xcami*, *scang*, *schang*).

${ }^{16}$ Voir l'article de Stefan Esders dans ce volume, ainsi que G. VISMARA, Ricerche sulla permuta nell'alto medioevo, in : ID., Scritti di storia giuridica II : La vita del diritto negli atti privati medievali (1987), p. 79-141.
} 
Les cooccurrences de commut* renvoient clairement au champ sémantique des transferts. Parmi les mots les plus fréquents se trouvent : vendendi (115), facerunt (90), donandi (87), donat (58), habendi (48), abendi (38), dedit (36), perdonandi (24), tenendi (33), mais aussi inter (182), se (137), liceat (87), terras (79), contra (47), terrarum (36), voluerit (36), primis (35), inprimis $(33)^{17}$.

Principales cooccurrences de commut*

\begin{tabular}{|c|c|c|c|c|c|}
\hline Ordre & $\begin{array}{lll}\text { Dans } & 5 & \text { mots } \\
\text { des deux côtés } & \end{array}$ & Ordre & $\begin{array}{l}\text { Dans } 5 \\
\text { du côté gauche }\end{array}$ & Ordre & $\begin{array}{l}\text { Dans } 5 \text { mots } \\
\text { du côté droit }\end{array}$ \\
\hline 1 & vendendi (115) & 1 & vendendi (108) & 1 & fecerunt (68) \\
\hline 2 & fecerunt (90) & 2 & liceat (83) & 2 & firma (40) \\
\hline 3 & liceat (87) & 3 & donandi (83) & 3 & permaneat (38) \\
\hline 4 & donandi (87) & 4 & terras (52) & 4 & ita (38) \\
\hline 5 & terras (79) & 5 & habendi (48) & 5 & terrarum (33) \\
\hline 6 & donat (58) & 6 & abendi (38) & 6 & terras (27) \\
\hline 7 & habendi (48) & 7 & tenendi (33) & 7 & facere (27) \\
\hline 8 & contra (47) & 8 & donat (33) & 8 & dedit (26) \\
\hline 9 & ita (41) & 9 & componat (28) & 9 & deberent (26) \\
\hline 10 & firma (40) & 10 & perdonandi (24) & 10 & voluerit (25) \\
\hline 11 & permaneat (38) & 11 & contra (23) & 11 & primis (25) \\
\hline 12 & abendi (38) & 12 & sive (22) & 12 & donat (25) \\
\hline 13 & voluerit (36) & 13 & hæc (22) & 13 & domnus (24) \\
\hline 14 & terrarum (36) & 14 & fecerunt (22) & 14 & contra (24) \\
\hline 15 & dedit (36) & 15 & seut (16) & 15 & ista (22) \\
\hline 16 & primis (35) & 16 & hec (16) & 16 & inprimis (22) \\
\hline 17 & tenendi (33) & 17 & quasdam (15) & 17 & villis (21) \\
\hline 18 & inprimis (33) & 18 & integrum (14) & 18 & partibus (20) \\
\hline 19 & facere (32) & 19 & suas (13) & 19 & rerum (19) \\
\hline 20 & componat (30) & 20 & auri (13) & 20 & faciendi (19) \\
\hline
\end{tabular}

Ces cooccurrences concernent, pour la plupart, des formules ou des syntagmes présents

\footnotetext{
${ }^{17}$ Les formes issues de comut* n'ont pas été prises en compte ici, mais aussi bien du point de vue de leur distribution chronologique que de leurs cooccurrences, elles suivent le même schéma que commut*. (comut* 29 formes, 94 occurrences : comutacione (1), comutacionem (3), comutaciones (1), comutacionis (2), comutad (1), comutamus (1), comutandas (1), comutandi (40), comutando (2), comutant (1), comutare (5), comutarent (2), comutat (3), comutatio (4), comutatione (2), comutationem (7), comutationes (2), comutationis (1), comutatores (1), comutatu (3), comutaverint (1), comutaverunt (1), comutavit (1), comutendi (1), comutentur (1), comutet (1), comutetur (1), comuto (3), comutores (1); dont 9 en 850-899, 28 en 900-949, 11 en 950-999, 4 en 1000-1049, 14 en 1050-1099, 28 sans date).
} 
dans différentes parties des actes, du dispositif aux clauses finales. La majorité des formes verbales et nominales les plus fréquentes de commut $^{*}$, apparaissent dès le début du IX ${ }^{\mathrm{e}}$ siècle. La plus ancienne ${ }^{18}$, et aussi la plus fréquente, dans l'ensemble envisagé ici, commutandi (161), figure dans les formules de nombreux actes de donation et de vente : ad habendum, vendendi, donandi, seu liceat commutandi ${ }^{19}$. Connue depuis au moins le VII $^{\mathrm{e}}$ siècle dans les diplômes mérovingiens, énumérant différentes actions de transfert pour affirmer le droit, y compris d'aliénation, sur le bien transféré, la formule concerne très peu d'actes d'échange ${ }^{20}$. Elle est utilisée, avec de nombreuses variantes et coalescences jusqu'au milieu du XI ${ }^{\mathrm{e}}$ siècle, réactualisée ponctuellement entre 1076 et 1083 avec des «nouveaux » types de transfert (precariandi ; livellandi ; impignorandi) ${ }^{21}$ pour se transformer encore ensuite ${ }^{22}$. La plupart des autres formes parmi les plus fréquentes - commutationem (118), commutare (86), commutatio (61), commutarent (57), commutatione (56), commutat (49), commutationis $(33)^{23}$, commutaverunt (28), commutant (22) - s'inscrivent en général dans des actes d'échange et dans des formules qui leur sont propres.

L' « effet formulaire », qui ressort bien à la lecture des occurrences les plus fréquentes, caractérise aussi les formes peu fréquentes à l'inverse de ce qu'on pourrait supposer en faisant l'hypothèse de leur présence hors-formulaire, dans des parties moins standardisées des actes. En plus de figurer en tant que variante à l'intérieur d'une formule récurrente, ces mots peu fréquents participent aussi à des formules plus rares elles aussi, mais formules quand même, comme le participe présent commutans (4) utilisé à trois reprises à Saint-Vincent de Mâcon au $\mathrm{X}^{\mathrm{e}}$ siècle dans pro transitoriis eterna commutans, et qui résonne à Cluny en 1065 dans transitoria stabilibus et temporalia eternis commutans ${ }^{24}$; ou comme la forme passive commutentur (2) employée deux fois à Cluny au $\mathrm{X}^{\mathrm{e}}$ siècle dans auctoritate scripturae pariter commutentur ${ }^{25}$.

Différentes expériences ont été réalisées avec les cooccurrences pour mieux préciser,

\footnotetext{
${ }^{18}$ Sav:153 (ca. 816).

${ }^{19}$ D'après Clu1:7 (833) (il s'agit d'une attribution de douaire).

${ }^{20}$ Quatre en tout : Clu1:49 (892), Macn:357 (899), Clu1:271 (926), Clu2:1254 (969).

${ }^{21}$ Clu4:3489 (1016), Clu4:3603 (1083), SAlb:259 (1081), Clu5:3658 (1091).

${ }^{22} \mathrm{Au} \mathrm{XIII}^{\mathrm{e}}$ siècle c'est tout un autre vocabulaire du transfert qui est utilisé, on dira plutôt : vendere, alienare, permutare seu pignori obligare - Yon3:649 (1269); concedere, permutare, obligare, distrahere, ac alias alienare - Bul:324 (1279).

${ }^{23}$ Les formes nominales en -cio - commutacionem (15), commutacio (12), commutacione (10), commutaciones (7), commutacionis (6), commutacionibus (2) - sont considérées ici séparément des variantes, plus fréquentes en -tio, mais ont été regroupées avec ces dernières dans les tableaux de cooccurrences réalisés à partir de la requête avec la troncature co[mn]muta[ct]io. *.

${ }^{24}$ Macn:73 (941), Macn:103 (950), Macn:455 (994), Clu4:3406 (1065).

${ }^{25}$ Clu1:249 (925), Clu2:1055 (958).
} 
dans le temps et selon les formes, verbales ou nominales, l'environnement lexical autour de commut*. Sans pouvoir entrer ici dans tous les détails de ces essais, notons d'abord que parmi les 64 formes issues de commut*, les formes verbales, actives et passives, sont plus nombreuses (46) que les formes nominales (18), mais sauf une exception, toutes les formes avec une seule occurrence, sont verbales, et sont aussi verbales les formes avec seulement deux (à l'exception de deux cas nominaux) ou trois occurrences.

Cooccurrences des formes nominales : entre mots supports et faisceaux de possibilités

Les tableaux de cooccurrences pour l'ensemble du corpus et par demi-siècle, extraits à partir de l'interrogation avec la troncature co[mn]muta[ct]io. * qui rassemble la plupart des formes nominales, permettent d'observer l'incidence ou pas des formulaires dans le temps. Les principales cooccurrences pour l'ensemble du corpus, dans l'intervalle de cinq mots avant et après co[mn]muta[ct]io.* (427) ${ }^{26}$, sont firma (39), permaneat (38), terrarum (34), componat (30), ista (24), voluerit (23), haec (22), contra (22), rerum (20), firmare (19), auri (19), hec (18), facta (18)... A l'exception de terrarum et rerum, utilisés par les éditeurs dans les titres ou analyses donnés aux chartes, les autres mots composent des formules présentes en fin d'acte, dans les clauses prohibitives et pénales (si quis contradicere voluerit, auri libras ii componat, et presens commutatio ista firma permaneat ${ }^{27}$; si quis vero contra hanc commutationem $^{28}$ ) ou dans l'eschatocole (qui commutacione ista fieri et firmare rogaverunt ${ }^{29}$ ). Ces formules, dont certains termes ou syntagmes sont utilisés depuis au moins la fin du VIII ${ }^{\mathrm{e}}$ siècle, se déclinent en de nombreuses variantes, mais les fréquences indiquent sur quels termes elles s'appuient. Vues par demi-siècle, il devient apparent que l'emploi de ces formules se situe au $\mathrm{X}^{\mathrm{e}}$ siècle, de même que le poids de celui-ci sur le tableau global. Les cooccurrences de co[mn]muta[ct]io. * entre 900-949 ${ }^{30}$ (110) donnent : firma (21), permaneat (20) componat (18), voluerit (15), auri (13), contra (11)...; et entre 950-999 (106) ${ }^{31}$ : terrarum (20), permaneat (16), firma (15), componat (10), villis (9), dant (8), stabilis (7),

\footnotetext{
${ }^{26}$ commutacio (12), commutacione (10), commutacionem (15), commutaciones (7), commutacionibus (2), commutacionis (6), commutatio (94), commutatione (73), commutationem (118), commutationes (27), commutationi (2), commutationibus (6), commutationis (55).

${ }^{27}$ Macn:244 (886).

${ }^{28}$ Clu1:259 (926).

${ }^{29}$ Clu1:49 (892).

${ }^{30}$ commutacio (8), commutacione (3), commutacionem (4), commutacionis (3), commutatio (31), commutatione (9), commutationem (45), commutationes (2), commutationi (1), commutationis (4).

31 commutacio (3), commutacione (1), commutacionem (2), commutacionis (1), commutatio (37), commutatione (19), commutationem (20), commutationes (9), commutationibus (1), commutationis (13).
} 
rerum (7), haec (7), dat (7), campum (7), voluerit (6)... Toujours en excluant les mots utilisés dans les titres et analyses des éditeurs des actes (terrarum, villis, rerum, dat, campum), on retrouve les mêmes mots support des formules finales.

Pour les autres siècles, la chose est moins évidente, soit parce que le nombre d'occurrences de co[mn]muta[ct]io. * sont trop peu nombreuses, comme dans la première moitié du IX ${ }^{\mathrm{e}}$ siècle (5), soit parce que la distribution des cooccurrences ne dégage pas de tendance principale, mais plutôt un faisceau de possibilités. Dans la deuxième moitié du IX siècle (23), si les cooccurrences rogaverunt (3), firmare (3), voluerit (2), permaneat (2), contra (2), componat (2), indiquent que les formules fréquentes du $\mathrm{X}^{\mathrm{e}}$ siècle étaient déjà employées, les autres, usibus (2), teneat (2), substractione (2), regio (2), praecepto (2), presens (2), minoratione (2), injusta (2), habere (2), deserviant (2) ${ }^{32}$, montrent qu'elles n'étaient pas dominantes. En fait, il n'y a que le démonstratif ista (6), des expressions commutatio/commutacione ista qui ressort relativement alors ${ }^{33}$.

Dans la première moitié du $\mathrm{XI}^{\mathrm{e}}$ siècle (39), on retrouve rogavit (3), firmare (3), rogaverunt (1), ce qui peut être la trace des formules fréquentes du $\mathrm{X}^{\mathrm{e}}$ siècle, à l'instar de ce que l'on observe dans la deuxième moitié du IX $\mathrm{e}^{\mathrm{e}}$, mais plus atténué encore. Ce qui change dans la première moitié du $\mathrm{XI}^{\mathrm{e}}$ siècle ce sont les cooccurrences qui indiquent des types de transferts. Toujours en excluant les mots des titres et analyses des éditeurs (terrarum, villis, vineam), on retrouve donacionem (4), titula[ct]ionem (3), quae (3), compara[ct]ionem (2), tradi[ct]iones (2), solidos (2), scribere (2), propria (2), rerum (2), notitia (2), cartulam (2)..., venditionis (1), venditio (1)..., c'est-à-dire, la tendance générale d'associer l'échange aux autres types de transferts, dans des actes qui ne concernent pas forcément un échange, comme dans les formules utilisées à Saint-Victor de Marseille (vel per donacionem seu per comparacionem, aut commutacionem, per titulacionem facimus donacionem ; firmissimam et inconvulsam commutacionem ut transfusam donacionem ${ }^{34}$ ), ou à Cluny (re conventio sive venditio aut donatio seu commutatio fit, posterorum memorice $e^{35}$. Cette tendance se confirme dans la deuxième partie du $\mathrm{XI}^{\mathrm{e}}$ siècle (17), avec les cooccurrences issues de donat* (4), de vendit* ${ }^{*}(2)$, ou encore transferre (1), tradiciones $(1)^{36}$, mais aussi posterorum (2) et felici (2),

\footnotetext{
${ }^{32}$ La plupart de ces mots renvoient à la formulation de deux actes d'Autun - sine aliqua substractione aut injusta minoratione vel commutatione usibus canonicorum sancti Nazarii deserviant - AuSM:5 (879), AuEc:I (887).

${ }^{33}$ Macn:244 (886), Clu1:49 (892).

${ }^{34}$ SVMa:4039 (1039), SVMa:4038 (1039), SVMg:511 (XI ${ }^{\mathrm{e}}$ s.).

${ }^{35}$ Clu4:3187 (1049).

36 aut vuerpitio, sive commutatio, memoriae posterorum - Clu4:334 (1050); prefatam donationem, commutationem, laudationem - SVMa:4165 (1051); venditionis aut commutationis vel excambiationis causa SVMa:4187 (1056); qui quaslibet donationes seu venditiones sive commutaciones agere temptaverit -
} 
qui font ressortir l'utilisation de commutatio dans l'acception de conversion de l'âme et des biens en vue de l'au-delà ${ }^{37}$. Notons tout de suite que c'est également dans cette acception que la forme au participe présent commutans apparaît à trois reprises au $\mathrm{X}^{\mathrm{e}}$ siècle dans la formule préambule pro transitoriis eterna commutans et une au $\mathrm{XI}^{\mathrm{e}}$ siècle dans temporalia eternis commutans $^{38}$.

$\mathrm{Au} \mathrm{XII}^{\mathrm{e}}$ siècle (32 et 40 occurrences de co[mn]muta[ct]io. ${ }^{*}$, pour la première et deuxième moitiés, respectivement), alors que les cooccurrences avec des termes indiquant les différents types de transferts se maintient, elles sont, à l'inverse du $\mathrm{XI}^{\mathrm{e}}$ siècle, plutôt des formes verbales issues de dare (dedi, dare, dedit, dedisse, dederunt, dederat, daturos), mais aussi de concedere (concessit, concedimus), de tradere (tradimus), de même que les formes nominales venditione et concessionis. Ces mots apparaissant en cooccurrence une ou deux fois seulement. On observe, en revanche, l'apparition d'un syntagme propre aux échanges à l'abbaye de La Ferté, dedit/fecerunt/fecit quandam commutationem, pour la première fois en 1113, mais employé surtout entre 1152 et $1162^{39}$.

Pour mettre en perspective ces observations issues des requêtes avec co[mn]muta[ct]io. *, la même expérience par demi-siècle a été réalisée avec une seule forme nominale, l'accusatif singulier commutationem, et sur l'ensemble du corpus avec les formes commutatio, commutatione, commutationis, commutationes. Sans pouvoir entrer dans le détail des résultats, notons simplement que les aspects généraux dégagés sur l'ensemble des formes nominales se confirment tout en se précisant pour chaque mot, notamment en ce qui concerne l'impact et la variété des formules au $\mathrm{X}^{\mathrm{e}}$ siècle.

Cooccurrences des formes verbales : terra générique

En ce qui concerne les formes verbales, actives et passives, l'effet formulaire est tout aussi sensible, mais à l'inverse des formes nominales, elles se situent au début des actes, dans le dispositif. Les expériences avec les cooccurrences ont été menées ici aussi sur tout le corpus et par demi-siècle, à partir d'une troncature d'interrogation - co[mn] mut.? [bmnrsv]*

Clu4:3404 (1065); kartam donationis vel commutacionis... donatione suprascripte commutationis firma et stabili - SVMa:4248 (1069); ut traditiones seu rerum commutationes cartis inserantur - Clu5:3686 (1094).

${ }^{37}$ Clu4:3364 (1060), Clu4:3377 (1061), Clu5:3670 (1093), Clu5:3676 (1094).

${ }^{38}$ Macn:78 (941), Macn:103 (950), Macn:355 (994), Clu4:3406 (1065).

${ }^{39}$ Fer:101 (1113), Fer:56 (1152), Fer:137 (1155) ; Fer:95 (1158), Fer:145 (1162). 
(489) - permettant de visualiser ensemble la plupart des formes verbales ${ }^{40}$, ainsi qu'à partir des formes individualisées commutandi, commutare, commutarent, commutat, commutaverunt, commutant. Sur l'ensemble du corpus, les principales cooccurrences de co[mn]mut.?[bmnrsv]* sont vendendi (116), liceat (87), donandi (87), terras (64), fecerunt (61), habendi (49), abendi (38), ita (35)... et elles prédominent également par demi-siècle entre 800 et 1099 avec une forte concentration entre 900 et 1049, rejoignant les résultats obtenus par l'interrogation avec la troncature commut*, c'est-à-dire pour l'ensemble des formes verbales et nominales.

Outre la prépondérance de la formule déjà citée de type ad habendum, vendendi, donandi, seu liceat commutandi, ressort aussi le formulaire propre aux échanges ut terras inter se commutarent, quod ita et fecerunt. Si l'expression inter se commut[asse] est employée dans des actes et diplômes au moins depuis le VIII ${ }^{\mathrm{e}}$ siècle $^{41}$ et à Saint-Vincent de Mâcon, depuis $825^{42}$, c'est l'introduction de l'accusatif pluriel terras qui semble ici un élément particulier dans la mesure où il s'agit du seul mot se référant à l'objet même des échanges entré dans un formulaire. A l'égard des précisions données par les actes, terra semble ici un terme générique, plus circonscrit que res qui est moins utilisé ${ }^{43}$, englobant toutefois les différents types de «terre»(campus, pratum, vinea...). Sous les formes $u t$ [quasdam] terras/terrulas inter se commutare[nt] ces expressions apparaissent presque une soixantaine de fois entre 886 et 1023 , à Saint-Vincent de Mâcon et à Cluny ${ }^{44}$, avec une nette concentration, plus de la moitié des cas, dans la deuxième moitié du $\mathrm{X}^{\mathrm{e}}$ siècle. Les cooccurrences des formes verbales commutat et commutant indiquent enfin l'utilisation de la

\footnotetext{
${ }^{40}$ commutabunt (1), commutamus (7), commutan (1), commutandi (161), commutandique (1), commutandis (1), commutando (5), commutandum (5), commutans (4), commutant (75), commutantes (4), commutare (87), commutarent (57), commutarentur (1), commutari (6), commutarint (1), commutarunt (5), commutasset (1), commutaveram (1), commutaverant (3), commutaverat (1), commutaverint (3), commutaverit (1), commutaveritis (1), commutaverunt (28), commutavi (6), commutavimus (4), commutavisse (3), commutavit (4), commutendi (1), commutendo (1), commutent (2), commutentur (2), commutores (1), conmutandi (1), conmutant (1), conmutaret (1), conmutavimus (1).

${ }^{41}$ Par exemple, le diplôme d'octobre 781 de Charlemagne à l'abbaye de Saint-Denis (Paris, Archives Nationales, $\mathrm{K} 7 \mathrm{n}^{\circ}$ 8/1 : http://www.cn-telma.fr/originaux/charte2954/ : res aliquas inter se commutasse.

${ }^{42}$ Macn:55 (825) - inter se commutare deberent; quod ita et fecerunt.

${ }^{43}$ Par exemple, res inter se commutarent et fecerunt - Macn:122 (887) ou ut res suas inter se commutarent; quod et fecerunt - Clu1:113:(910) et leurs variantes, apparaissent une quinzaine de fois à Mâcon et à Cluny, entre 887 et 988.

${ }^{44}$ Macn:240 (886), Clu3:2783 (1023). Dans les originaux conservés en France l'expression apparaît à la même époque aussi à Nouaillé - Poitiers, Archives départementales de la Vienne, C $8 \mathrm{n}^{\circ} 10$ (886) - http://www.cntelma.fr/originaux/charte1067 (ut terras eorum inter se commutar[e de]berent). A noter les différentes variantes, comme ut de terris eorum inter se commutare - Clu1:220 (920), ou ut aliquid de terris suis inter se commutarent - Clu1:612 (942), etc. Sur le rôle-clé du scriptorium de Saint-Vincent de Mâcon dans la mise en place des formulaires à Cluny, voir N. PERrEAuX, Dynamique sociale et écriture documentaire (Cluny, $\mathrm{X}^{\mathrm{e}}-\mathrm{XII}^{\mathrm{e}}$ siècle). Observations statistiques sur le champ sémantique de l'eau, in : Cluny, le monachisme et la société au premier âge féodal (880-1050), dir. D. IOGNA-PRAT et alii (à paraître).
} 
formule dona[n]t atque [adque/et/sive/vel] commuta[n]t 46 fois, entre la fin du IX et le début $\mathrm{du} \mathrm{XI}^{\mathrm{e}}$ siècle ${ }^{45}$, parfois en association avec le formulaire ut terras inter se commutarent... (18).

\section{Cambiare et permutare : pragmatique vernaculaire et synonymie lettrée}

Des expériences similaires ont été effectuées avec les formes du groupe ${ }^{*} c a m b i{ }^{*}+$ (*cambi*, concami*, *scami*, *xcami*, *scang*, *schang*), qui rassemble un total de 174 formes différentes. Ici, les formes verbales et nominales ont été traitées ensemble. Les principales cooccurrences - fecerunt (52), dos (33), nous (32), voluerit (23), nom (23), fecit (23), terras (21), permutacion (19)... - font apparaître des mots latins et des mots français anciens. Les premiers se retrouvent aussi parmi les principales cooccurrences de commut*, les seconds, comme montre l'interrogation par demi-siècle, sortent des formulaires de la deuxième moitié du XIII ${ }^{\mathrm{e}}$ siècle, à l'exception de dos qui est une notation d'éditeur («au dos »), pour indiquer les mentions dorsales des originaux de Cluny. Ces mentions dorsales médiévales désignent un échange surtout par les mots escamium (1), scamium (31), scammium (15), scamnium (6), scamnum (3) ${ }^{46}$.

Principales cooccurrences de *cambi*+ $\left({ }^{*}{ }^{*}\right.$ cambi ${ }^{*}$, concami $^{*},{ }^{*}$ scami*, *xcami*, ${ }^{*}$ scang*, *schang*)

\begin{tabular}{|c|c|c|c|c|c|}
\hline Ordre & $\begin{array}{lcc}\text { Dans } & 5 & \text { mots } \\
\text { des deux côtés } & \end{array}$ & Ordre & $\begin{array}{l}\text { Dans } 5 \\
\text { du côté gauche }\end{array}$ & Ordre & \begin{tabular}{|lcl} 
Dans & 5 & mots \\
du côté droit &
\end{tabular} \\
\hline 1 & fecerunt (52) & 1 & $\operatorname{dos}(33)$ & 1 & fecerunt (51) \\
\hline 2 & $\operatorname{dos}(33)$ & 2 & nous (21) & 2 & fecit (22) \\
\hline 3 & nous (32) & 3 & terras (20) & 3 & voluerit (18) \\
\hline 4 & ou (25) & 4 & ou (15) & 4 & primis (17) \\
\hline 5 & voluerit (23) & 5 & locum (14) & 5 & permutacion (14) \\
\hline 6 & nom (23) & 6 & o (13) & 6 & firmare (13) \\
\hline 7 & fecit (23) & 7 & lettres (12) & 7 & permaneat (12) \\
\hline 8 & terras (21) & 8 & $\operatorname{cop}(12)$ & 8 & nom (12) \\
\hline 9 & permutacion (19) & 9 & avons (12) & 9 & facere (12) \\
\hline 10 & li (19) & 10 & nom (11) & 10 & nous (11) \\
\hline
\end{tabular}

\footnotetext{
${ }^{45}$ Macn:240 (886) et Clu3:2571 (1003), pour les premières et dernières occurrences. Ces formules sont en partie introduites par les expressions adverbiales in primis (6), inprimis (10), e contra (1), econtra (2).

${ }^{46}$ Par exemple, la mention dorsale Scamium Ademari in Colonicas dans 1'acte Clu2:1612 (982). Sur les mentions dorsales des chartes de Cluny, voir S. BARRET, La mémoire et l'écrit: l'abbaye de Cluny et ses archives $\left(\mathrm{X}^{\mathrm{e}}\right.$ XVIII $^{\mathrm{e}}$ siècle) (2004), p. 123-132, 140-141, 318-330.
} 


\begin{tabular}{ll|lll|l|l|l|l|l|l|}
$\mathbf{1 1}$ & $\operatorname{choses}(19)$ & $\operatorname{terram}(10)$ & li (11) \\
\hline
\end{tabular}

Les cooccurrences latines les plus fréquentes indiquent deux tendances, particulièrement fortes au $\mathrm{X}^{\mathrm{e}}$ siècle, jusqu'au début $\mathrm{du} \mathrm{XI}^{\mathrm{e}}$ siècle. D'une part, l'utilisation des dérivés de cambio/cambiare dans certains formulaires déjà repérés avec les dérivés de commutatio/commutare, comme avec la cooccurrence terras et fecerunt: ut terras inter se scamiarent, quod ita fecerunt ${ }^{47}$; ou voluerit dans les formules pénales finales : hoc scamium calumpniari voluerit, auri libras ii componat ${ }^{48}$. D'autre part, des formules propres aux dérivés de cambio, qui ressortent avec les cooccurrences fecerunt et fecit, dans les formules Scamium fecerunt $(23)^{49}$ ou Scamium fecit $(11)^{50}$ (dont 28 occurrences au $\mathrm{X}^{\mathrm{e}}$ siècle). Ce type de formule, utilisée en début d'actes courts qui notent l'essentiel de l'action réalisée, n'apparaît pas avec commutationem. Il en va de même avec les expressions in loco/locum escamio/scamii...(34), employées surtout et également au $\mathrm{X}^{\mathrm{e}}$ siècle $(26)^{51}$. Tout ceci, au regard aussi de la préférence donnée à scamium dans les mentions dorsales médiévales, pose la question des limites de la synonymie, ou plutôt de l'usage parallèle, entre commutatio et scamio et du choix du terme vernaculaire dans les usages les plus pratiques.

Entre le $\mathrm{XI}^{\mathrm{e}}$ et la première moitié du XIII ${ }^{\mathrm{e}}$ siècle, on retrouve une situation de faisceau de possibilités similaire à celle observée pour commutatio au $\mathrm{XI}^{\mathrm{e}}$ siècle : des fréquences basses, et par conséquent multiples, de cooccurrences (parfois 7 ou 6 , mais surtout 3 et 2 fois). Mais si les cooccurrences désignant d'autres types de transfert sont perceptibles au $\mathrm{XI}^{\mathrm{e}}$ et dans la première moitié du XIII ${ }^{\mathrm{e}}$ siècle, aucune tendance ne se détache au XII ${ }^{\mathrm{e}}$.

\footnotetext{
${ }^{47}$ Par exemple, dans Clu2:1396 (974).

${ }^{48}$ Par exemple, dans Clu1:744 (949).

${ }^{49}$ Par exemple, dans Clu1:309 (927) (Scamium fecerunt monachi Clunienses cum Gauzerius cum uxore sua Ingelburgis. In primis donat Gauzerius et Ingelburgis in villa Cucugnis campum unum; habet perticas in longum XXXII, et in latum V; qui terminat de tribus parte terra Sancti Petri, et de quarto parte via publica. Econtra donant monachi supradicto viro Gauzerius et uxore sua Ingelburgis donamus campum unum; haget in longo perticas XXVIIII, in latus habet perticas IIII; et terminat de una parte terra Eva, et alia parte cuta currentem, de tertia parte ipso Gauzerius; et alia peciola in ipsa villa Villarellio; terminat de una parte ipso Gauzerius, de alia parte via publica, et de tertia parte terra Sancti Petri, et de quarta parte terra Adaleldis; et alium servicium mansum unum debet Bercenno. Et faciant unusquisque quicquid facere voluerint. S. Gauzerius et uxor sua Ingelburgis. S. Letaldus. S. Gunzolinus. S. Ubertus. S. Bernardus.)

${ }^{50}$ Par exemple, dans Clu2:923 (954) (Scamium fecit Fredelo cum monachis. In primis donat Fredelo unum campum monachis; qui terminat a medio die via publica, a mane terra comptalis, a sero terra Arlulf, a cercio rivo volvente. Est autem ipse campus in villa Curconcingas. Et in ipsa villa donant monachi jam dicto viro duas peciolas de terra, quarum et terminat una ex eis a mane et a medio die terra Sancti Vincencii, a cercio terra comptalis, a mane terra Tedbaldi; alia peciola terminat de una parte terra Flotberti, de alia parte terra Sancti Vincentii. S. Fredelo. S. Costabilis. S. Ermenardi. S. Constancii.)

${ }^{51}$ Par exemple, Clu1:69 (900), Macn:119 (930). Un seul exemple de l'emploi de l'expression in loco commutationis a été repéré, et il date du $\mathrm{XI}^{\mathrm{e}}$ siècle - Clu4:2856 (1035).
} 
Les cooccurrences de ${ }^{*} c a m b i *+$ en ancien français nous amènent à la deuxième moitié du XIII ${ }^{\mathrm{e}}$ et au début XIV ${ }^{\mathrm{e}}$ siècle. Parmi les nombreuses observations possibles, on remarquera seulement l'emploi de choses (19), de façon générique et l'absence d'un autre terme plus précis pour désigner ce qui est échangé ; on n'est plus dans la généralisation de terra. Les expressions en latin ou en ancien français permutationem seu escambium ${ }^{52}$ ou en nom deschange et de permutacion ${ }^{53}$, introduisent quant à elles l'association (synonymique ?) avec permutatio au détriment de commutatio. Il faut souligner que les expressions assimilables à la synonymie sont cependant relativement peu récurrentes dans l'ensemble du corpus, 21 fois pour les dérivés de commutatio/cambio, 19 pour permutatio/cambio, et qu'elles se concentrent au $\mathrm{X}^{\mathrm{e}}$ siècle pour les premières et dans la deuxième moitié du XIII ${ }^{\mathrm{e}}$ pour les secondes. Le sens de ces associations semble toutefois différent, permutatio apparaissant comme mot «nouveau » et pourtant de référence, récupéré du vocabulaire juridique du latin classique, en parallèle aux termes courants vernaculaires. Les principales cooccurrences de permut* $^{*}$ sur l'ensemble du corpus ${ }^{54}$, titulo (21), dicto (16), causa (16), indiquent dans la deuxième partie du XIII ${ }^{\mathrm{e}}$ siècle, la mise en place de toute une nouvelle gamme de formules, et de mots, comme titulo permutationis tradiderint et concesserint, ou in litteris super dicto contractu seu tractatu permutationis, ou encore ex causa permutationis ${ }^{55}$.

\section{En guise de conclusion : détour par la Patrologie latine}

Récapitulant et schématisant les observations réalisées sur le corpus des chartes bourguignonnes, on constate du point de vue du nombre d'occurrences deux moments forts, le $\mathrm{X}^{\mathrm{e}}$ et la deuxième moitié du $\mathrm{XIII}^{\mathrm{e}}$ siècle. Ces deux moments sont chacun liés à l'emploi de termes latins particuliers, commutatio/commutare au $\mathrm{X}^{\mathrm{e}}$, permutatio/permutare au XIII ${ }^{\mathrm{e}}$. Ces termes sont utilisés en parallèle avec les mots vernaculaires issus de cambio/cambiare, qui constituent l'élément lexical constant sur toute la période, pour lequel nous avons fait l'hypothèse de l'usage pragmatique de mise en registre d'un transfert : les formules dans des actes d'échange concis et les notes dorsales médiévales étant les principaux indices. L'interrogation avec Philologic sur l'ensemble de la Patrologie latine apporte un élément supplémentaire dans ce même sens. La requête avec *ambio*+ ${ }^{*}$ cambi $^{*}$, concami $^{*}$,

\footnotetext{
${ }^{52}$ Par exemple, Clu6:5050 (1262).

${ }^{53}$ Par exemple, Hug:455 (1289).

${ }^{54}$ En laissant toujours de côté les termes employés par les éditeurs dans leurs analyses : terrarum (62), villis (38), rerum (17)...

${ }^{55}$ Par exemple, et respectivement, dans Hug:274:(1272), AuEp2:92:(1295), Yon3:572:(1257).
} 
${ }^{*}$ scami $^{*}, *_{x c a m i}{ }^{*},{ }^{*}$ scang $*{ }^{*}$ schang $\left.{ }^{*}\right)(590)^{56}$ fait ressortir que les différentes formes se trouvent dans des textes diplomatiques ou dans des compositions qui les reprennent ou en font référence, comme dans les annales, les chroniques ou les gestae. Les principales cooccurrences - episcopo (14), quidquid (13), loco (13), dedit (13), terram (12).. proviennent ainsi des diplômes et des bulles des $\mathrm{XI}^{\mathrm{e}}$ et $\mathrm{XII}^{\mathrm{e}}$ siècles ${ }^{57}$, et sont associées, pour la plupart, aux formes issues de concambio. Ces occurrences viennent confirmer la spécialisation des dérivés de cambio pour rendre compte des échanges, et leur emploi de préférence dans des textes de type pragmatique.

L'enquête sur permut* (1262) dans la Patrologie, fait apparaître la multiplicité d'acceptions de permutatio/permutare qui mériterait une étude serrée comme celle menée par Isabelle Rosé sur commutatio. Les occurrences nombreuses dans les textes juridiques, les bulles et diplômes des $\mathrm{XI}^{\mathrm{e}}$ et $\mathrm{XII}^{\mathrm{e}}$ siècles, semblent cependant pointer ces documents comme l'un des relais possibles de l'emploi de permutatio pour désigner l'échange, avant qu'il ne s'introduise et ne se généralise dans les chartes bourguignonnes au XIII ${ }^{\mathrm{e}} \operatorname{siècle}^{58}$.

Les principales cooccurrences de commut*, comut*, conmut*(6026) dans la Patrologie ${ }^{59}$ - melius (375), anima (112), veritatem (111), episcopum (109), finem (102), ambrichonem (100), mendacium (95), deterius (93), gloriam (88), absque (87), illis (83), dabit (80), facta (78)... - renvoient en premier lieu à l'emploi récurrent et dans différents contextes de l'expression in melius commutare (326) et, en moindre mesure à son opposé in deterius commutare (77). Ensuite, les passages bibliques - d'abord ceux des Psaumes, rappelées par l'expression in finem, pro his qui commutabuntur [ipsi David] $(80)^{60}$, suivis de ceux des Romains $1,25^{61}$, Isaie 55, $1^{62}$, Matthieu 16, 26 et Marc $8,37^{63}$ et Osée 4, $7^{64}$ - pèsent tout autant que les 96 actes d'échange de l'évêque Ambrichon de Ratisbonne (864-891) dans le Liber traditionum de Saint-Emmeran du $\mathrm{IX}^{\mathrm{e}}$ siècle $^{65}$. Comme dans les actes, les différentes

\footnotetext{
${ }^{56}$ Cette requête fait apparaître aussi des formes absentes des chartes, mais qui ne dérivent pas de cambio et ne désignent pas l'échange (obliviscor, reminiscor, etc.). Ainsi les cooccurrences les plus fréquentes - dixi (28), hora (27), venerit (26) - sont liées à reminiscamini (42) dans le passage de l'Evangile de Jean (16, 4) : sed haec locutus sum vobis ut cum venerit hora eorum reminiscamini quia ego dixi vobis.

57 Par exemple, Lucius III [1181-1185], Epistola et privilegia, Migne PL 129 c. 1105A : quam concambio diversarum terrarum.

${ }^{58}$ Par exemple, Henricus IV imperator [1050-1106], Diplomata, MIGNE PL 151 c. 1093D : de permutatione facta.

${ }^{59}$ Sur les différentes acceptions de la commutatio dans la Patrologie, voir I. Rose, Commutatio, passim.

${ }^{60}$ Ps 44,$1 ; 68,1 ; 79,1$.

${ }^{61}$ qui commutaverunt veritatem Dei in mendacium (76 occurrences).

${ }^{62}$ venite emite absque argento et absque ulla commutatione vinum et lac (65 occurrences)

${ }^{63}$ aut quam dabit hommo commutationem pro anima sua (55 occurrences).

${ }^{64}$ gloriam eorum in ignominiam commutabo (50 occurrences).

${ }^{65}$ Migne PL 129 c. 899-962 (Commutatio inter Ambrichonem episcopum...).
} 
formes issues de commutatio présentes dans d'autres genres de texte, se caractérisent aussi par leur emploi syntagmatique et formulaire, la Bible étant un «recueil de formules » par excellence.

Ce qui paraît distinguer l'emploi de commutatio, cambio, permutatio et leurs dérivés dans les actes c'est leur proximité avec des mots désignant d'autres types de transfert, et cela sur la longue durée. Que ce soit la copule commutat/donat, les juxtapositions habendum, vendendi, donandi, commutandi au $\mathrm{X}^{\mathrm{e}}$ siècle, ou vendere, alienare, permutare seu pignori obligare au XIII ${ }^{\mathrm{e}}$ siècle, l'échange se conjugue à l'intérieur d'une gamme. C'est donc à au sein d'un ensemble qu'il semble trouver cohérence. Et il ne s'agit pas de simple tautologie, car si les sondages réalisés avec les formes issues de venditio ou donatio pointent aussi un « effet formulaire », parmi leurs cooccurrences les plus fréquentes ne se trouvent pas a priori d'autres types de transferts, omniprésents pourtant dans les cooccurrences des termes de l'échange. De même, la fracture entre la fin du $\mathrm{X}^{\mathrm{e}}$ et le début du $\mathrm{XI}^{\mathrm{e}}$ siècle, avec la chute des mentions des formes dérivées de commutatio, et vraisemblablement des actes d'échange, tendance qui se vérifie un peu partout en Occident comme le montrent plusieurs contributions de ce volume, semble aussi un changement dans la mise en registre des transferts avec la création de nouvelles formules et d'agencements lexicaux. Ceci après une période de très forte réitération d'anciennes formules mais aussi de création d'expressions spécialisées, introduisant des notions à la fois génériques et circonscrites, comme terra à la place de res, et utilisant les formes vernaculaires dérivées de cambio dont l'emploi demeure stable. Cela ne signifie pas forcément qu'on n'échange plus, mais que la gamme des transferts se dit autrement par écrit, qu'il y a mutation. Il y a aussi mutation au XIII ${ }^{\mathrm{e}}$ siècle, avec le « retour» de l'échange et l'introduction de permutatio. Ces observations n'ont cependant qu'une valeur partielle, dans la mesure où c'est l'articulation de toute la palette des transferts soumis au dénominateur commun de la donation qu'il faut comprendre, à commencer par la mise en parallèle de l'échange et de la vente/achat qui relèvent d'un même système de « réciprocité exigible » : on aurait ainsi un profil sans doute différent du XII ${ }^{\mathrm{e}}$ siècle, voire du $\mathrm{XI}^{\mathrm{e}}$, que la recherche sur les seuls termes de l'échange laisse dans l'ombre. Il n'empêche qu'en faisant l'expérience d'une « lecture à distance », les formules et les expressions qui se dégagent des principales cooccurrences des mots désignant l'échange, apparaissent moins comme des stéréotypes que comme des structures nodales agissantes, où se réalisent les moments et les éléments-clé des mutations dans les modes de dire et d'écrire les transferts. 


\section{Annexes}

Recueils d'actes utilisés

\begin{tabular}{|c|c|c|c|}
\hline $\begin{array}{l}\text { Nombre } \\
\text { d'actes }\end{array}$ & Lieu & Edition & abréviation \\
\hline \multicolumn{4}{|c|}{ BOURGOGNE } \\
\hline 4 & Auberive & $\begin{array}{l}\text { Ch. ROYER, Chartes concernant l'abbaye d'Auberive, in Bulletin } \\
\text { de la Société historique et archéologique de Langres, } 2 \text { (1886), p. } \\
124-130 .\end{array}$ & Aub \\
\hline 344 & Autun & $\begin{array}{l}\text { A. de CHARMASSE, Cartulaire de l'Eglise d'Autun, Paris, 1865- } \\
1900 .\end{array}$ & $\mathrm{AuEc}$ \\
\hline 328 & Autun & A. de CHARMASSE, Cartulaire de l'Evêché d'Autun, Paris, 1880. & $\mathrm{AuEv}$ \\
\hline 101 & Autun & $\begin{array}{l}\text { J.-G. Bulliot, Essai historique sur l'abbaye de Saint-Martin } \\
\text { d'Autun, de l'ordre de saint Benoît, Autun, } 1849 .\end{array}$ & AuSM \\
\hline 39 & Beaujeu & $\begin{array}{l}\text { M.-C. GUIGUE, Cartulaire de l'église collégiale Notre-Dame de } \\
\text { Beaujeu, Lyon, } 1864 .\end{array}$ & Bea \\
\hline 25 & Bellary & $\begin{array}{l}\text { L. CHARRAULT, La chartreuse de Bellary (1209-1793), in Bulletin } \\
\text { de la Société nivernaise des lettres, sciences et arts, } 22 \text { (1908), p. } \\
\text { 541-632. }\end{array}$ & Bell \\
\hline 642 & Chalon & $\begin{array}{l}\text { B. PROST., S. BOUGENOT, Cartulaire de Hugues de Chalon (1220- } \\
\text { 1319), Lons-le-Saunier, } 1904 \text { (Publications historiques et } \\
\text { archéologiques de la Société d'émulation du Jura). }\end{array}$ & Hug \\
\hline 119 & Chalon & $\begin{array}{l}\text { P. CANAT DE CHIZY, Cartulaire du prieuré de Saint-Marcel-lès- } \\
\text { Chalon, Chalon-sur-Saône, Marceau, } 1894 .\end{array}$ & StMar \\
\hline 173 & $\begin{array}{l}\text { Charité- } \\
\text { sur-Loire }\end{array}$ & $\begin{array}{l}\text { R. de LESPINASSE, Cartulaire du prieuré de la Charité-sur-Loire } \\
\text { (Nièvre), ordre de Cluni, Nevers, } 1887 .\end{array}$ & Char \\
\hline 445 & Cluny & $\begin{array}{l}\text { P. SIMON, Bullarium sacri Ordinis cluniacensis, complectens } \\
\text { plurima privilegia per summos pontifices tum ipsi cluniacensi } \\
\text { abbatiae, tum ei subditis monasteriis hactenus concessa, Lyon, } \\
\text { Jullieron, } 1680\end{array}$ & Bul \\
\hline 884 & Cluny & $\begin{array}{l}\text { A. BERNARD, A. BRUEL, Recueil des chartes de l'abbaye de } \\
\text { Cluny, vol. 1, Paris, } 1876\end{array}$ & Clu1 \\
\hline 848 & Cluny & $\begin{array}{l}\text { A. BERNARD, A. BRUEL, Recueil des chartes de l'abbaye de } \\
\text { Cluny, vol. 2, Paris, } 1880\end{array}$ & Clu2 \\
\hline 1069 & Cluny & $\begin{array}{l}\text { A. BERNARD, A. BRUEL, Recueil des chartes de l'abbaye de } \\
\text { Cluny, vol. 3, Paris, } 1884\end{array}$ & Clu3 \\
\hline 864 & Cluny & $\begin{array}{l}\text { A. BERNARD, A. BRUEL, Recueil des chartes de l'abbaye de } \\
\text { Cluny, vol. } 4,1888\end{array}$ & Clu4 \\
\hline 814 & Cluny & $\begin{array}{l}\text { A. BERNARD, A. BRUEL, Recueil des chartes de l'abbaye de } \\
\text { Cluny, vol. 5, } 1894\end{array}$ & Clu5 \\
\hline 1095 & Cluny & $\begin{array}{l}\text { A. BERNARD, A. BRUEL, Recueil des chartes de l'abbaye de } \\
\text { Cluny, vol. } 6,1903\end{array}$ & Clu6 \\
\hline 27 & Corbigny & $\begin{array}{l}\text { A. de CHARMASSE, Chartes de l'abbaye de Corbigny, Autun, } \\
1889 .\end{array}$ & Corb \\
\hline 270 & $\begin{array}{l}\text { Ferté-sur- } \\
\text { Grosne }\end{array}$ & $\begin{array}{l}\text { G. DUBY, Recueil des pancartes de l'abbaye de la Ferté-sur- } \\
\text { Grosne : 1113-1178, Gap : Impr. Louis Jean, } 1953 \text { (réimpression } \\
\text { anastatique, Bruxelles, 2000, Bibliothèque du Moyen Age, 17) }\end{array}$ & $\mathrm{Ff}$ \\
\hline 173 & $\begin{array}{l}\text { Jully-les- } \\
\text { Nonnains }\end{array}$ & $\begin{array}{l}\text { E. PETIT, Cartulaire du prieuré de Jully-les-Nonnains, Auxerre, } \\
1881\end{array}$ & Jull \\
\hline 638 & Mâcon & $\begin{array}{l}\text { M.C. RAGUT, Cartulaire de Saint-Vincent de Mâcon : connu sous } \\
\text { le nom de Livre enchaîné, Mâcon, } 1864 .\end{array}$ & Macn \\
\hline 22 & $\begin{array}{l}\text { Mèves-sur- } \\
\text { Loire }\end{array}$ & $\begin{array}{l}\text { R. de LESPINASSE, Titres du prieuré de Mèves-sur-Loire, XIIe- } \\
\text { XVIII }{ }^{\text {e }} \text { s., in Bulletin de la Société Nivernaise des Lettres, } \\
\text { Sciences et Arts, } 7 \text { (1899), p. 196-198. }\end{array}$ & Mvv \\
\hline 139 & Mores & $\begin{array}{l}\text { Ch. LALORE, Chartes de l'abbaye de Mores, Troyes, } 1873 \\
\text { (Mémoires de la Société académique de l'Aube, 37, p. 5-112). }\end{array}$ & Mor \\
\hline 128 & Nevers & $\begin{array}{l}\text { R. de LespinAsSE, Cartulaire de Saint-Cyr de Nevers, Paris, } \\
\text { 1916. }\end{array}$ & $\mathrm{NvSC}$ \\
\hline
\end{tabular}




\begin{tabular}{|c|c|c|c|}
\hline 57 & Nevers & $\begin{array}{l}\text { R. de LESPINASSE, Les chartes de Saint-Etienne de Nevers, in } \\
\text { Bulletin de la Société nivernaise des lettres, sciences et arts, } 22 \\
\text { (1907), p. 51-130 }\end{array}$ & NvSE \\
\hline 246 & $\begin{array}{l}\text { Paray-le- } \\
\text { Monial }\end{array}$ & $\begin{array}{l}\text { U. CHEVALIER, Chartularium prioratys beatae mariae de Paredo } \\
\text { monachorum, Montbéliard, } 1891\end{array}$ & ParM \\
\hline 5 & Tannay & $\begin{array}{l}\text { H. de FlAMARE, Les anciennes chartes de la collégiale de } \\
\text { Tannay, Paris, 1891, 8 p. (Extrait du Bulletin historique et } \\
\text { philologique du Comité des Travaux Historiques et Scientifiques, } \\
\text { 1891, p. 1-8). }\end{array}$ & Tan \\
\hline 5 & Tonnerre & $\begin{array}{l}\text { M. JOLIVOT, Chartes de l'église Saint-Pierre de Tonnerre, Bulletin } \\
\text { de la société des sciences historiques et naturelles de l'Yonne, } 36 \\
\text { (1882), p. 191-194. }\end{array}$ & Ton \\
\hline 243 & Tournus & $\begin{array}{l}\text { P. JUENIN, Nouvelle Histoire de l'abbaye royale et collégiale de } \\
\text { Saint-Filibert et de la ville de Tournus, Dijon, } 1733 \text {, Preuves. }\end{array}$ & Torn \\
\hline 10 & Val-Suzon & $\begin{array}{l}\text { G. DESJARDINS, Cartulaire de l'abbaye de Conques en Rouergue, } \\
\text { Paris, } 1879, n^{\circ} 445-447,458,483-484,488-490 \text { et } 539 .\end{array}$ & Val \\
\hline 385 & Yonne & $\begin{array}{l}\text { M. QUANTIN, Cartulaire général de l'Yonne, vol. 1, Auxerre, } \\
1854-1860\end{array}$ & Yon1 \\
\hline 526 & Yonne & $\begin{array}{l}\text { M. QUANTIN, Cartulaire général de l'Yonne, vol. 2, Auxerre, } \\
1854-1860\end{array}$ & Yon2 \\
\hline 742 & Yonne & $\begin{array}{l}\text { M. QUANTIN, Cartulaire général de l'Yonne, Supplément, } \\
\text { Auxerre, } 1873 \text {. }\end{array}$ & Yon3 \\
\hline \multicolumn{4}{|c|}{ VALLÉE DU RHÔNE et PROVENCE } \\
\hline 189 & Ainay & $\begin{array}{l}\text { A. BERNARD, Cartulaire de l'Abbaye de Savigny, suivi du Petit } \\
\text { cartulaire de l'Abbaye d'Ainay, } 2 \text { vol., Paris, } 1853\end{array}$ & Ain \\
\hline 969 & Savigny & $\begin{array}{l}\text { A. BERNARD, Cartulaire de l'Abbaye de Savigny, suivi du Petit } \\
\text { cartulaire de l'Abbaye d'Ainay, } 2 \text { vol., Paris : Impr. impériale, } \\
1853\end{array}$ & Sav \\
\hline 276 & Vienne & $\begin{array}{l}\text { U. CHEVALIER, Cartulaire de l'abbaye de Saint-André-Le-Bas-de- } \\
\text { Vienne, ordre de Saint Benoît, suivi d'un Appendice de chartes } \\
\text { inédites sur le diocèse de Vienne (IX }{ }^{\mathrm{e}} \text {-XII }{ }^{\mathrm{e}} \text { siècles), Lyon, N. } \\
\text { Scheuring, } 1869\end{array}$ & SAlb \\
\hline 346 & Lérins & $\begin{array}{l}\text { H. MORIS, E. BLANC, Cartulaire de l'abbaye de Lérins, vol. 1, } \\
\text { Paris, } 1883\end{array}$ & Ler \\
\hline 331 & Marseille & $\begin{array}{l}\text { Actes originaux de Saint-Victor de Marseille (d'après la base des } \\
\text { originaux de l'ARTEM) }\end{array}$ & SVMa \\
\hline 953 & Marseille & $\begin{array}{l}\text { B. GUERARD, Cartulaire de l'abbaye de Saint-Victor de Marseille, } \\
2 \text { vol., Paris, C. Lahure, } 1857\end{array}$ & SVMg \\
\hline 14474 & & TOTAL & \\
\hline
\end{tabular}

